\title{
Behavioral health risk factor profiles in general hospital patients: identifying the need for screening and brief intervention
}

Jennis Freyer-Adam ${ }^{1,2^{*}}$ D, Florian Noetzel ${ }^{3}$, Sophie Baumann ${ }^{2,4,5}$, Ali Alexander Aghdassi ${ }^{6}$, Ulrike Siewert-Markus ${ }^{1,2}$, Beate Gaertner ${ }^{7}$ and Ulrich John ${ }^{2,5}$

\begin{abstract}
Background: Little is known about the clustering of behavioral health risk factors (HRFs), namely the occurrence of 16 specific combinations of tobacco smoking, at-risk alcohol use, overweight and physical inactivity in general hospital patients. Furthermore, social inequalities in HRFs, health and life expectancy are a major concern in public health. In order to establish the need for screening and intervention in general hospital care, the study aimed to determine the co-occurrence of HRFs in patients in four medical departments, and to investigate differences by gender, age and socio-economic characteristics.
\end{abstract}

Methods: Over 17 months, a systematic multiple HRF screening was conducted at one general hospital in northeastern Germany. In total, 6251 18-64 year old patients (92\% of eligibles) participated. Proportions and confidence intervals were calculated for all 16 HRF profiles stratified by department, gender, age group, school education, and employment status.

Results: In total, $92.2 \%$ of the participants (58.6\% male) reported $\geq 1 \mathrm{HRF}$, and $65.7 \% \geq 2 \mathrm{HRFs}$. Men (71.2\%), patients aged 35-49 (67.9\%) and 50-64 years (69.5\%), lower educated (79.0\%), and unemployed (77.8\%) patients had larger proportions of $\geq 2$ HRFs than their counterparts. In all departments, the most common HRF profiles included overweight. HRF profiles that included alcohol and/ or smoking were more common in ear-nose-throat and trauma surgery than in internal medicine and general surgery patients. Men had higher rates concerning almost all HRF profiles including $\geq 2 \mathrm{HRFs}$ and alcohol; women concerning profiles that included $\leq 2 \mathrm{HRFs}$ and inactivity. In older patients, profiles with $\geq 2 \mathrm{HRFs}$ including overweight; and in younger patients, profiles with smoking and/or alcohol were more common. In lower educated patients, profiles with $\geq 2 \mathrm{HRFs}$ including inactivity; and in higher educated patients profiles with $\leq 2 \mathrm{HRFs}$ including alcohol were more common. Compared to others, unemployed patients had higher rates of profiles with $\geq 3$ HRFs including smoking.

Conclusions: Two in three patients require interventions targeting two or more HRFs. The findings help to develop screening and brief intervention for patients with specific health risk profiles, that can reach most patients, including those most in need and those most hard to reach, with socio-economically disadvantaged people in particular.

Registry: clinicaltrials.gov: NCT01291693.

Keywords: Risk factors, Health behaviors, Smoking, Alcohol, Physical inactivity, Overweight, General hospital, Health care, Prevention, Chronic diseases

\footnotetext{
* Correspondence: freyer@uni-greifswald.de

'Institute for Medical Psychology, University Medicine Greifswald,

Walther-Rathenau-Str. 48, 17475 Greifswald, Germany

${ }^{2}$ German Center for Cardiovascular Research, Site Greifswald, Fleischmannstr.

42-44, 17475 Greifswald, Germany

Full list of author information is available at the end of the article
}

(c) The Author(s). 2019 Open Access This article is distributed under the terms of the Creative Commons Attribution 4.0 International License (http://creativecommons.org/licenses/by/4.0/), which permits unrestricted use, distribution, and reproduction in any medium, provided you give appropriate credit to the original author(s) and the source, provide a link to the Creative Commons license, and indicate if changes were made. The Creative Commons Public Domain Dedication waiver (http://creativecommons.org/publicdomain/zero/1.0/) applies to the data made available in this article, unless otherwise stated. 


\section{Introduction}

Modifiable behavioral health risk factors (HRFs), particularly tobacco smoking, at-risk alcohol use, unbalanced diet and physical inactivity, are major contributors to the development of non-communicable diseases and to all-cause deaths [1-3]. HRFs inhibit successful recovery or improved wellbeing and increase mortality after diagnosis as found for cancer patients (e.g. $[4,5])$. To prevent and treat prevalent non-communicable diseases, abstaining from tobacco smoking, keeping body weight within the healthy range, being physically active as part of everyday life, and limiting alcohol consumption is recommended [2, 6-8].

The energy-balance related HRFs, i.e. physical inactivity and either unhealthy diet or overweight; as well as the two substance-use related HRFs smoking and at-risk alcohol use are intertwined and often cluster $[9,10]$. However, a total of 16 specific combinations of the four major modifiable HRFs are possible and observed in the general population [11-13]. About half of the general population in high-income countries report multiple, i.e. two or more of the four HRFs; and even larger proportions are found when insufficient vegetable and fruit intake is considered as an indicator of unhealthy diet instead of overweight [11-14]. The EPIC-Norfolk prospective population study revealed strong trends of increasing mortality with an increasing number of HRFs, particularly cardiovascular causes of death [15]. A protective effect on mortality risk was found with each additional health recommendation met [16]. Furthermore, co-occurring HRFs may not only have an additive but more than a multiplicative effect on disease incidence and/ or mortality as was found for example for alcohol and smoking concerning various cancers $[17,18]$.

Gender, age and socio-economic status (SES) are related to the accumulation of HRFs and HRF profiles. A systematic review revealed a greater number of HRFs in men than in women, while findings concerning age were rather mixed with some studies showing more HRFs among younger and other studies showing more HRFs among older people [19]. More risky HRF clusters were also found in people with lower levels of education [19]. Social inequalities in life expectancy and health between persons at the bottom and those at the top of the social scale are a major concern in public health, and the accumulation of HRFs such as alcohol and smoking has been suggested to explain these differences [13, 20-22]. Recent findings suggest that SES may be even more relevant than gender in the accumulation of the four HRFs [23].

To address behavioral HRFs in people, the general hospital has been found to be particularly suitable. Hospitalization itself may be a health event that might motivate individuals to change unhealthy behaviors $[24,25]$. Increased patient motivation to change HRFs was found for smokers and alcohol dependent patients $[24,26,27]$, and could provide a "window of opportunity" for brief behavior change interventions [26]. Among a sample of adult general hospital patients in the United Kingdom who participated in a survey postdischarge, more than $80 \%$ considered the hospital a good location for receiving health education on the HRFs, and agreed that all patients should be asked about HRFs [28]. Brief interventions developed to be implemented in health care often target single HRFs. They have been found to be effective in altering single behaviors [29-37] and in improving measures of health $[31,38]$. However, interventions targeting multiple HRFs may be more cost-efficient and effective in preventing or treating non-communicable diseases as various interdependent issues may be addressed (e.g. $[19,39])$. Encouraging findings on the efficacy of such interventions applied in the general population and general practice setting have been reported [40-43].

Given the significance of HRFs in the development of chronic diseases [1], HRF profiles including either one or more of the four HRFs might be expected to be particularly common in general hospital inpatients. However, systematically drawn data is scarce. A systematic screening study that assessed five behavioral HRFs (the four plus low vegetable and fruit intake) and depressive symptoms at three sites in Germany revealed that $83 \%$ of the patients screened positive for two or more of the six screening targets [44]. HRF profiles and their variation across gender, age and SES have not been investigated in the general hospital. Furthermore, little is known about whether hospital departments vary in occurrence of multiple HRFs in their patients, and whether different screening and intervention foci may be justified or needed to address different HRFs or HRF profiles.

The aims of the study were: 1 . To determine the cooccurrence of four behavioral HRFs (i.e. tobacco smoking, at-risk alcohol use, overweight, physical inactivity) and their co-occurrence in $16 \mathrm{HRF}$ profiles among patients attending a general hospital. 2. To describe and to compare HRF profiles across four major medical departments (i.e. general medicine, general surgery, trauma surgery, ear-nose-throat). 3. To describe and compare HRF profiles by gender, age and variables related to SES (i.e. school education, employment status). The results will be useful to show the need for interventions targeting multiple HRFs, and to design a comprehensive screening and intervention protocol.

\section{Methods}

Data were obtained as part of the screening procedure for the randomized controlled trial "Testing delivery channels of individualized motivationally tailored alcohol interventions among general hospital patients: in-person versus computerbased, PECO” (ClinicalTrials.gov: NCT01291693). As described in more detail elsewhere $[45,46]$, the trial tested the comparative two-year efficacy of two ways of delivering brief 
interventions, namely in-person counseling and computergenerated written feedback, targeting the single HRF at-risk alcohol use [38, 47-49].

\section{Sampling frame and participants}

Over 17 consecutive months in 2011 and 2012, participants were recruited in the four major departments at the University Medicine Hospital Greifswald in Germany: internal medicine (endocrinology, nephrology, cardiology, gastroenterology, angiology, pneumology), general surgery (general and thorax surgery), trauma surgery, and ear-nose-throat. All wards of each department, except intensive care units, were included, resulting in 13 wards. The hospital provides general hospital care for 600,000 inhabitants in Greifswald and surrounding communities.

All patients aged 18-64 years admitted to one of the four departments were approached on weekdays, by one of three research assistants. Patients were asked to complete a self-administrated questionnaire on health behaviors using an electronic handheld device. Patients cognitively $(n=21)$ or physically incapable or terminally ill $(n=159)$, with highly infectious diseases $(n=101)$, discharged or transferred outside the study area within the first $24 \mathrm{~h}$ or within the weekend on which they were admitted $(n=627)$, already screened for the study during an earlier hospital stay $(n=2779)$, with insufficient German language skills $(n=93)$, or employed at the conducting research institute $(n=4)$ were excluded. In total, 6809 of 10,593 patients assessed met screening inclusion criteria. Of these, 414 were missed before discharge or transferal, 107 declined participation, 36 provided insufficient data, and 1 died, leaving 6251 (91.8\%) participants who provided informed oral consent and sufficient data.

\section{Measurements \\ Behavioral HRFs}

Four HRFs were assessed: two substance-use related HRFs (smoking, alcohol at-risk drinking) and two energy-balance related HRFs (physical inactivity, overweight). Smoking was assessed using the question "Are you a tobacco smoker currently?" and four response categories differentiating between current daily smoking, occasional smoking, former smoking and never smoking. Current occasional or daily smoking was considered as HRF. Alcohol at-risk drinking was determined using the total score of the Alcohol Use Disorder Identification Test-Consumption (range: 0-12) [50]. Alcohol at riskdrinking was considered present in females and males with scores of $\geq 4$ and $\geq 5$, respectively. These recommended gender-specific cut-offs had shown good sensitivity and specificity in detecting at-risk alcohol use including but not limited to alcohol use disorders [51], and correspond well to the national limits defined for healthy people, i.e. $>12 / 24 \mathrm{~g}$ of pure alcohol per day and $>$ 3/ 4 drinks per occasion for women/ men [52]. Overweight was assessed using the body-mass-index obtained by self-reported weight in kilogram and height in meters. A body-mass-index $\geq 25.0$ [53] was defined as overweight. Physical inactivity was measured by asking "Do you also do sports? "with six response categories: none, $<1,1-2$, $2-3,3-4$ and $>4 \mathrm{~h}$ per week. As at least $75 \mathrm{~min}$ of vigorous- or $150 \mathrm{~min}$ of moderate-intensity physical activity per week are recommended [54,55], participants with none or less than $1 \mathrm{~h}$ were considered inactive.

\section{Grouping variables}

Medical department (internal medicine, general surgery, trauma surgery, earn-nose-throat) was recorded. Gender (male, female) was assessed. Participants were allocated to three age groups: 18-34, 35-49, 50-64 years. SES included school education and employment status. To determine highest school education achieved, various German school types were categorized as a) lowest level including 9 years of school or less, b) medium level including 10 to 11 years of school, and c) highest level including 12 or more years of school. Employment status distinguished between currently employed, unemployed, and other. "Other" included retired persons (69.2\%), students (12.6\%), housewives $(6.1 \%)$, and not further specified (12.1\%).

\section{Statistical analyses}

Proportions and 95\% confidence intervals (CI) for multiple, i.e. two or more HRFs, and for each of the $16 \mathrm{HRF}$ profiles are given for the total sample; and separately for each medical department, gender, age group, level of school, and employment status. The 16 HRF profiles included one healthy profile (no HRF), four single factor profiles (smoking, alcohol, overweight or inactivity [only]), six profiles with two HRFs (smoking plus alcohol, smoking plus overweight, smoking plus inactivity, alcohol plus overweight, alcohol plus inactivity, overweight plus inactivity), four profiles with three HRFs (smoking plus alcohol plus overweight, smoking plus alcohol plus inactivity, smoking plus overweight plus inactivity, alcohol plus overweight plus inactivity), and one profile with all four HRFs (smoking plus alcohol plus overweight plus inactivity). Non-overlapping CIs were considered statistically significant, with two CIs just touching indicating significant differences at about $p<0.01$ [56].

In addition, the mean number of HRFs and standard deviation (SD) are given for the total sample and separately for each subgroup. To investigate variables associated with HRF number (counts: 0, 1, 2, 3, 4), a multivariate poisson regression analysis with medical department, gender, age group, level of school, and employment status as predictors was calculated. $P$-values $<0.05$ were considered 
statistically significant. Cases with missing values were excluded list-wise. STATA version 13.1 SE was used.

\section{Results}

\section{Sample characteristics}

The sample was on average 45.7 years old $(\mathrm{SD}=13.3)$. With observed numbers given in Tables $1,49.4 \%$ of the sample were aged 50 years or older, $58.6 \%$ were male, $21.8 \%$ had the lowest level of school education, 59.2\% were employed, and $36.4 \%$ were recruited on the internal medicine department.

\section{Single HRFs}

Inactivity was the most common HRF (65.6\%), followed by overweight $(61.0 \%)$, smoking $(39.0 \%)$, and at-risk alcohol use (henceforth alcohol, 21.2\%). As indicated by non-overlapping 95\% CIs in Table 1, statistically significantly differences in single HRFs were found across different medical departments, socio-demographic and socio-economic subgroups $(p<0.01)$. While a larger proportion of trauma surgery and in part ear-nosethroat patients reported smoking (44.5, $44.1 \%$ versus $33.8,34.2 \%$ ) and alcohol (25.6, $23.2 \%$ versus $21.2,18.8 \%$ ), a larger proportion of internal medicine and in part general surgery patients reported inactivity $(71.7,67.4 \%$ versus $57.3,64.4 \%)$ and overweight $(64.6,61.7 \%$ versus 58.9 , $56.7 \%$, Table 1). Compared to women, a larger proportion of men reported smoking (42.6\% versus $33.8 \%$ ), alcohol (27.9\% versus $11.7 \%)$ and overweight $(66.0 \%$ versus $53.8 \%$ ). The older the age group was, the lower was the proportion that reported smoking $(50.4 \%$ versus $43.9 \%$ versus $30.7 \%)$ and alcohol $(28.1 \%$ versus $22.2 \%$ versus $17.3 \%$ ), and the higher the occurrence of inactivity $(50.3 \%$ versus $68.5 \%$ versus $71.7 \%)$ and overweight (41.7\% versus $63.3 \%$ versus $69.4 \%$ ). The higher the level of school education was, the lower was the occurrence of smoking (51.0\% versus $38.4 \%$ versus $26.6 \%$ ), inactivity (78.1\% versus $66.6 \%$ versus $47.4 \%$ ) and/ or overweight (65.7, 63.2\% versus $48.5 \%$ ), but the occurrence of alcohol was higher in patients with the highest compared to those with medium level of school education $(24.7 \%$ versus 19.7\%). A larger proportion of unemployed patients reported smoking (48.6\% versus 37.5, 36.6\%), alcohol ( $28.8 \%$ versus $22.2,16.0 \%)$ and inactivity $(75.7 \%$ versus $61.5,70.0 \%$ ) than employed and other patients, respectively.

Table 1 Occurrence of each behavioral health risk factor in the total sample and stratified by medical department, gender, age and socio-economics

\begin{tabular}{|c|c|c|c|c|c|c|c|c|c|}
\hline \multirow[t]{2}{*}{ Subgroups } & \multirow[t]{2}{*}{ Number } & \multicolumn{2}{|c|}{ Smoking } & \multicolumn{2}{|c|}{ Alcohol } & \multicolumn{2}{|c|}{ Inactivity } & \multicolumn{2}{|c|}{ Overweight } \\
\hline & & $\%$ & $(95 \%$ Cl) & $\%$ & $(95 \%$ Cl) & $\%$ & $(95 \%$ Cl) & $\%$ & $(95 \%$ Cl) \\
\hline Total & 6251 & 39.0 & $(37.7-40.2)$ & 21.2 & $(20.2-22.2)$ & 65.6 & $(64.4-66.8)$ & 61.0 & $(59.7-62.2)$ \\
\hline \multicolumn{10}{|l|}{ Medical department } \\
\hline Internal medicine & 2284 & 33.8 & (31.9-35.8) & 21.2 & $(20.2-22.2)$ & 71.7 & $(69.8-73.6)$ & 64.6 & $(62.6-66.6)$ \\
\hline General surgery & 957 & 34.2 & $(31.1-37.3)$ & 18.8 & $(17.2-20.5)$ & 67.4 & $(64.3-70.4)$ & 61.7 & $(58.5-64.7)$ \\
\hline Ear-nose-throat & 1282 & 44.1 & $(41.4-46.9)$ & 23.2 & $(21.0-25.7)$ & 64.4 & $(61.7-67.0)$ & 56.7 & $(53.9-59.4)$ \\
\hline Trauma surgery & 1728 & 44.5 & $(42.1-46.9)$ & 25.6 & $(23.5-27.7)$ & 57.3 & $(55.0-59.7)$ & 58.9 & $(56.5-61.2)$ \\
\hline \multicolumn{10}{|l|}{ Gender } \\
\hline Men & 3665 & 42.6 & $(41.0-44.2)$ & 27.9 & $(26.5-29.4)$ & 65.3 & $(63.7-66.8)$ & 66.0 & $(64.5-67.6)$ \\
\hline Women & 2586 & 33.8 & $(32.0-35.7)$ & 11.7 & $(10.5-13.0)$ & 66.0 & $(64.1-67.8)$ & 53.8 & $(51.8-55.7)$ \\
\hline \multicolumn{10}{|l|}{ Age in years } \\
\hline $18-34$ & 1546 & 50.4 & $(47.9-52.9)$ & 28.1 & $(25.9-30.5)$ & 50.3 & $(47.7-52.8)$ & 41.7 & $(39.2-44.2)$ \\
\hline $35-49$ & 1615 & 43.9 & $(41.5-46.4)$ & 22.2 & $(20.2-24.3)$ & 68.5 & $(66.2-70.7)$ & 63.3 & $(60.9-65.7)$ \\
\hline $50-64$ & 3090 & 30.7 & $(29.0-32.2)$ & 17.3 & $(16.0-18.7)$ & 71.7 & $(70.1-73.3)$ & 69.4 & $(67.7-71.0)$ \\
\hline \multicolumn{10}{|c|}{ School education achieved } \\
\hline Lowest level & 1354 & 51.0 & $(48.3-53.7)$ & 22.3 & $(20.1-24.6)$ & 78.1 & (75.8-80.3) & 65.7 & $(63.1-68.2)$ \\
\hline Medium level & 3715 & 38.4 & $(36.8-39.9)$ & 19.7 & $(18.5-21.1)$ & 66.6 & $(65.1-68.1)$ & 63.2 & $(61.6-64.8)$ \\
\hline Highest level & 1137 & 26.6 & $(24.0-29.2)$ & 24.7 & $(22.2-27.3)$ & 47.4 & $(44.5-50.4)$ & 48.5 & $(45.5-51.4)$ \\
\hline \multicolumn{10}{|l|}{ Employment } \\
\hline Unemployed & 749 & 52.2 & $(48.6-55.8)$ & 28.8 & $(25.6-32.2)$ & 75.7 & $(72.5-78.7)$ & 62.8 & $(59.2-66.2)$ \\
\hline Employed & 3680 & 37.5 & $(35.9-39.0)$ & 22.2 & $(20.8-23.5)$ & 61.5 & $(59.9-63.0)$ & 61.1 & $(59.5-62.6)$ \\
\hline Other & 1791 & 36.6 & $(34.4-38.9)$ & 16.0 & $(14.3-17.7)$ & 70.0 & $(67.8-72.1)$ & 60.0 & $(57.7-62.3)$ \\
\hline
\end{tabular}

Notes: $\mathrm{Cl}=$ confidence interval, non-overlapping $95 \% \mathrm{Cls}$ indicate significant differences at $p \leq 0.01$ 
HRF profiles and medical department

Overall, the mean HRF number was $1.9 \quad(\mathrm{SD}=1.0$, Table 2); $92.2 \%$ of the patients reported at least one and $65.7 \%$ multiple, i.e. two or more, HRFs. In the total sample and in eight of 15 subgroups, the three most common HRF profiles were overweight plus inactivity (22.4\%), overweight (11.6\%) and smoking plus overweight plus inactivity (10.7\%). Having no HRF was ranked 6th among the 16 HRF profiles (7.8\%).

Patients from different medical departments did not differ significantly concerning the occurrence of any and multiple HRFs (Table 2). Concerning the HRF profiles, five HRF profiles, each involving smoking and/ or alcohol were significantly more common in trauma surgery (smoking, alcohol, smoking plus alcohol, smoking plus overweight, smoking plus alcohol plus overweight) and partly in ear-nose-throat patients (smoking, alcohol, smoking plus alcohol) than in internal medicine patients (Table 2). Smoking plus alcohol was also more common in trauma surgery patients than in general surgery patients. Two energy-balance related HRF profiles were significantly more common in internal medicine than in trauma surgery patients (inactivity), and in internal medicine and general surgery patients than in ear-nose- throat and trauma surgery patients (overweight plus inactivity).

\section{HRF profiles, gender and age}

A significantly larger proportion of men reported any and multiple (71.2\% versus $57.9 \%)$ HRFs than women (Table 3). Six of the 15 risky HRF profiles, each with multiple HRFs and alcohol (smoking plus alcohol, alcohol plus overweight, smoking plus alcohol plus overweight, smoking plus alcohol plus inactivity, alcohol plus overweight plus inactivity, and all four HRFs), were significantly more common in men. Three frequent HRF profiles, each involving inactivity and a maximum of two HRFs (inactivity, smoking plus inactivity, overweight plus inactivity) were more common in women.

A significantly larger proportion of both older age groups (i.e. 35-49 and 50-64 year olds) reported any and multiple $(69.5,67.9 \%$ versus $57.3 \%)$ HRFs than the youngest age group (18-35 year olds, Table 3$)$. While having no HRF was the most common profile in the youngest age group (12.8\%), seven of the 15 risky HRF profiles, each involving smoking and/ or alcohol, were significantly more common in either the youngest (smoking, alcohol, smoking plus alcohol, smoking plus

Table 2 Occurrence of multiple health risk factors and of each behavioral health risk factor profile in the total sample and stratified by medical department

\begin{tabular}{|c|c|c|c|c|c|c|c|c|c|c|}
\hline \multirow{2}{*}{$\begin{array}{l}\text { Health risk factor(s) } \\
\text { Mean number (standard deviation) }\end{array}$} & \multicolumn{2}{|c|}{$\begin{array}{l}\text { Total } \\
(n=6251)\end{array}$} & \multicolumn{2}{|c|}{$\begin{array}{l}\text { Internal medicine } \\
(n=2284)\end{array}$} & \multicolumn{2}{|c|}{$\begin{array}{l}\text { General surgery } \\
(n=957)\end{array}$} & \multicolumn{2}{|c|}{$\begin{array}{l}\text { Ear-nose-throat } \\
(n=1282)\end{array}$} & \multicolumn{2}{|c|}{$\begin{array}{l}\text { Trauma surgery } \\
(n=1728)\end{array}$} \\
\hline & 1.9 & $(1.0)$ & 1.9 & $(0.9)$ & 1.8 & $(0.9)$ & 1.9 & $(1.0)$ & 1.9 & $(1.0)$ \\
\hline & $\%$ & $(95 \%$ Cl) & $\%$ & $(95 \%$ Cl) & $\%$ & $(95 \%$ Cl) & $\%$ & $(95 \%$ Cl) & $\%$ & $(95 \%$ Cl) \\
\hline Multiple, $\geq 2$ & 65.7 & $(64.5-66.9)$ & 67.4 & $(65.5-69.3)$ & 64.6 & $(61.5-67.6)$ & 65.5 & $(62.8-68.1)$ & 64.2 & $(61.9-66.5)$ \\
\hline \multicolumn{11}{|l|}{ Profile } \\
\hline no health risk factor & 7.8 & $(7.1-8.5)$ & 5.7 & $(4.8-6.7)$ & 8.7 & $(7.0-10.6)$ & 8.3 & $(6.9-10.0)$ & 9.6 & $(8.3-11.1)$ \\
\hline smoking (only) & 3.7 & $(3.3-4.2)$ & 2.4 & $(1.8-3.1)$ & 3.2 & $(2.2-4.6)$ & 4.7 & $(3.6-6.0)$ & 5.0 & $(4.1-6.2)$ \\
\hline alcohol (only) & 1.4 & $(1.2-1.8)$ & 0.8 & $(0.5-1.30)$ & 1.6 & $(0.9-2.6)$ & 1.8 & $(1.1-2.7)$ & 1.9 & $(1.32-2.7)$ \\
\hline overweight (only) & 11.6 & $(10.8-12.4)$ & 12.7 & $(11.4-14.2)$ & 11.4 & $(9.4-13.6)$ & 9.8 & $(8.3-11.6)$ & 11.5 & $(10.0-13.1)$ \\
\hline inactivity (only) & 9.8 & $(9.0-10.5)$ & 10.9 & $(9.7-12.3)$ & 10.6 & $(8.7-12.7)$ & 9.8 & $(8.3-11.6)$ & 7.7 & $(6.5-9.1)$ \\
\hline smoking plus alcohol & 1.9 & $(1.6-2.3)$ & 1.0 & $(0.6-1.51)$ & 0.9 & $(0.4-1.8)$ & 2.3 & $(1.52-3.2)$ & 3.3 & $(2.5-4.3)$ \\
\hline smoking plus overweight & 4.0 & $(3.5-4.5)$ & 2.8 & $(2.1-3.5)$ & 3.6 & $(2.5-4.9)$ & 4.4 & $(3.4-5.7)$ & 5.6 & $(4.6-6.8)$ \\
\hline smoking plus inactivity & 8.8 & $(8.1-9.5)$ & 8.7 & $(7.5-9.9)$ & 8.5 & $(6.8-10.4)$ & 10.5 & $(8.9-12.3)$ & 7.9 & $(6.6-9.2)$ \\
\hline alcohol plus overweight & 2.5 & $(2.1-2.9)$ & 2.1 & $(1.6-2.8)$ & 2.0 & $(1.2-3.1)$ & 2.5 & $(1.7-3.5)$ & 3.2 & $(2.4-4.1)$ \\
\hline alcohol plus inactivity & 1.4 & $(1.1-1.7)$ & 1.6 & $(1.1-2.2)$ & 1.8 & $(1.0-2.8)$ & 1.2 & $(0.7-2.0)$ & 1.0 & $(0.6-1.6)$ \\
\hline overweight plus inactivity & 22.4 & $(21.4-23.4)$ & 27.6 & $(25.8-29.5)$ & 27.0 & $(24.2-29.9)$ & 18.2 & $(16.1-20.4)$ & 16.0 & $(14.3-17.8)$ \\
\hline smoking plus alcohol plus overweight & 1.5 & $(1.2-1.9)$ & 0.7 & $(0.4-1.2)$ & 1.3 & $(0.6-2.2)$ & 1.8 & $(1.1-2.7)$ & 2.5 & $(1.8-3.3)$ \\
\hline smoking plus alcohol plus inactivity & 4.3 & $(3.8-4.8)$ & 4.3 & $(3.5-5.2)$ & 3.1 & $(2.1-4.4)$ & 4.6 & $(3.5-5.9)$ & 4.7 & $(3.8-5.9)$ \\
\hline smoking plus overweight plus inactivity & 10.7 & $(10.0-11.5)$ & 10.4 & $(9.2-11.7)$ & 10.8 & $(8.9-12.9)$ & 10.9 & $(9.2-12.8)$ & 11.1 & $(9.6-12.6)$ \\
\hline alcohol plus overweight plus inactivity & 4.2 & $(3.8-4.8)$ & 4.6 & $(3.8-5.5)$ & 2.9 & $(2.0-4.2)$ & 4.1 & $(3.1-5.4)$ & 4.6 & $(3.6-5.7)$ \\
\hline all four health risk factors & 4.0 & $(3.5-4.5)$ & 3.6 & $(2.9-4.5)$ & 2.8 & $(1.9-4.1)$ & 4.9 & $(3.8-6.2)$ & 4.4 & $(3.5-5.5)$ \\
\hline
\end{tabular}

Notes: $\mathrm{Cl}=$ confidence interval, non-overlapping $95 \% \mathrm{Cls}$ indicate significant differences at $\mathrm{p} \leq 0.01$ 
Table 3 Occurrence of multiple health risk factors and of each behavioral health risk factor profile stratified by gender and age

\begin{tabular}{|c|c|c|c|c|c|c|c|c|c|c|}
\hline \multirow{2}{*}{$\begin{array}{l}\text { Health risk factor(s) } \\
\text { Mean number (standard deviation) }\end{array}$} & \multicolumn{2}{|c|}{$\begin{array}{l}\text { Women } \\
(n=2586)\end{array}$} & \multicolumn{2}{|c|}{$\begin{array}{l}\text { Men } \\
(n=3665)\end{array}$} & \multicolumn{2}{|c|}{$\begin{array}{l}18-34 \text { years } \\
(n=1546)\end{array}$} & \multicolumn{2}{|c|}{$\begin{array}{l}35-49 \text { years } \\
(n=1615)\end{array}$} & \multicolumn{2}{|c|}{$\begin{array}{l}50-64 \text { years } \\
(n=3090)\end{array}$} \\
\hline & 1.7 & $(0.9)$ & 2.0 & $(1.0)$ & 1.7 & $(1.0)$ & 2.0 & (1.0) & 1.9 & (0.9) \\
\hline & $\%$ & (95\% Cl) & $\%$ & (95\% Cl) & $\%$ & (95\% Cl) & $\%$ & $(95 \%$ Cl) & $\%$ & $(95 \%$ Cl) \\
\hline Multiple, $\geq 2$ & 57.9 & $(56.0-59.8)$ & 71.2 & $(69.7-72.7)$ & 57.3 & $(54.8-59.8)$ & 69.5 & $(67.2-71.8)$ & 67.9 & $(66.3-69.6)$ \\
\hline \multicolumn{11}{|l|}{ Profile } \\
\hline no health risk factor & 10.9 & $(9.7-12.2)$ & 5.6 & $(4.8-6.4)$ & 12.8 & $(11.2-14.6)$ & 6.7 & $(5.6-8.1)$ & 5.8 & $(5.0-6.7)$ \\
\hline moking (only) & 4.0 & $(3.3-4.8)$ & 3.5 & $(2.9-4.2)$ & 8.2 & $(6.9-9.7)$ & 3.1 & $(2.30-4.1)$ & 1.8 & $(1.3-2.31)$ \\
\hline alcohol (only) & 1.4 & $(0.9-1.9)$ & 1.5 & $(1.1-1.9)$ & 3.9 & $(3.0-5.0)$ & 0.9 & $(0.5-1.5)$ & 0.5 & $(0.3-0.8)$ \\
\hline overweight (only) & 11.3 & $(10.1-12.6)$ & 11.8 & $(10.8-12.9)$ & 8.3 & $(7.0-9.8)$ & 9.9 & $(8.5-11.5)$ & 14.1 & $(12.9-15.4)$ \\
\hline inactivity (only) & 14.5 & $(13.2-16.0)$ & 6.4 & $(5.6-7.2)$ & 9.4 & $(8.0-11.0)$ & 9.8 & $(8.4-11.4)$ & 9.9 & $(8.8-11.0)$ \\
\hline smoking plus alcohol & 1.0 & $(0.7-1.5)$ & 2.5 & $(2.0-3.1)$ & 5.3 & $(4.2-6.5)$ & 1.2 & $(0.76-1.9)$ & 0.5 & $(0.3-0.84)$ \\
\hline smoking plus overweight & 3.3 & $(2.6-4.0)$ & 4.5 & $(3.9-5.3)$ & 5.8 & $(4.6-7.0)$ & 5.3 & $(4.3-6.5)$ & 2.5 & $(1.9-3.1)$ \\
\hline smoking plus inactivity & 10.2 & $(9.0-11.4)$ & 7.8 & $(7.0-8.7)$ & 10.9 & $(9.4-12.6)$ & 8.7 & $(7.3-10.1)$ & 7.8 & $(6.9-8.8)$ \\
\hline alcohol plus overweight & 1.5 & $(1.0-2.0)$ & 3.2 & $(2.6-3.8)$ & 2.7 & $(1.9-3.6)$ & 2.6 & $(1.9-3.5)$ & 2.3 & $(1.8-2.9)$ \\
\hline alcohol plus inactivity & 1.0 & $(0.7-1.5)$ & 1.6 & $(1.2-2.1)$ & 1.8 & $(1.21-2.6)$ & 0.7 & $(0.3-1.22)$ & 1.5 & $(1.1-2.0)$ \\
\hline overweight plus inactivity & 24.3 & $(22.6-26.0)$ & 21.0 & $(19.7-22.4)$ & 8.8 & $(7.4-10.3)$ & 20.9 & $(18.9-22.9)$ & 30.0 & $(28.4-31.6)$ \\
\hline smoking plus alcohol plus overweight & 0.7 & $(0.4-1.1)$ & 2.1 & $(1.7-2.6)$ & 2.8 & $(2.0-3.7)$ & 1.7 & $(1.16-2.5)$ & 0.8 & $(0.5-1.15)$ \\
\hline smoking plus alcohol plus inactivity & 3.2 & $(2.6-4.0)$ & 5.0 & $(4.4-5.8)$ & 6.0 & $(4.8-7.2)$ & 5.5 & $(4.4-6.7)$ & 2.8 & $(2.3-3.5)$ \\
\hline smoking plus overweight plus inactivity & 9.8 & $(8.7-11.0)$ & 11.4 & $(10.4-12.5)$ & 7.6 & $(6.3-9.0)$ & 13.4 & $(11.8-15.1)$ & 10.9 & $(9.9-12.1)$ \\
\hline alcohol plus overweight plus inactivity & 1.3 & $(0.9-1.8)$ & 6.3 & $(5.6-7.2)$ & 1.9 & $(1.3-2.7)$ & 4.6 & $(3.6-5.7)$ & 5.2 & $(4.5-6.1)$ \\
\hline all four health risk factors & 1.7 & $(1.2-2.2)$ & 5.6 & $(4.9-6.4)$ & 3.9 & $(3.0-5.0)$ & 5.0 & $(3.9-6.1)$ & 3.5 & $(2.9-4.2)$ \\
\hline
\end{tabular}

Notes: $\mathrm{Cl}=$ confidence interval, non-overlapping $95 \% \mathrm{Cls}$ indicate significant differences at $\mathrm{p} \leq 0.01$

inactivity) or in both younger age groups, i.e. the 18-34 and 35-49 year olds (smoking plus overweight, smoking plus alcohol plus overweight, smoking plus alcohol plus inactivity). Four HRF profiles, each involving overweight, were significantly more common in either the oldest compared to both younger groups (overweight) or among both older groups compared to the youngest (smoking plus overweight plus inactivity, alcohol plus overweight plus inactivity). The occurrence of the most common HRF profile overweight plus inactivity increased significantly across age groups $(8.8 \%$ versus $20.9 \%$ versus $30.0 \%$ ).

\section{HRF profiles, school education and employment status}

The lower the level of school was, the significantly larger the proportion of any and multiple HRFs $(79.0 \%$ versus $66.5 \%$ versus $48.0 \%$, Table 4 ). While having no HRF was the most common profile in the group with the highest level of school (16.9\%), six of the 15 risky HRF profiles, each involving a single HRF or two HRFs including alcohol, were also significantly more common in groups with higher school levels: either in the group with the highest level compared to the two groups with lower levels (alcohol, smoking plus alcohol), in the two groups with higher levels compared to the group with the lowest level (overweight, inactivity), or in the group with the highest level compared to the group with the lowest level (alcohol plus overweight, alcohol plus inactivity). Five HRF profiles, each involving two or more HRFs including inactivity, were significantly more common in groups with lower school level: either in the group with the lowest level compared to the two groups with higher levels (smoking plus alcohol plus inactivity), in the two groups with lower levels compared to the group with the highest level (overweight plus inactivity), or occurrence decreased significantly with each level of school (smoking plus inactivity, smoking plus overweight plus inactivity, and all four HRFs).

A significantly larger proportion of unemployed patients reported any and multiple ( $77.8 \%$ versus $63.6,65.3 \%)$ HRFs than employed and other patients (Table 4). Three HRF profiles, each involving three or all four HRFs and smoking were significantly more common in unemployed than in employed (smoking plus alcohol plus inactivity, smoking plus overweight plus inactivity, all four HRFs) and other patients (smoking plus alcohol plus inactivity, all four HRFs). Two HRF profiles were significantly more common in employed than in unemployed (overweight) or other patients (alcohol plus overweight plus inactivity). Two HRF profiles (inactivity, overweight plus inactivity) were significantly more common in others than in the employed and unemployed. 
Table 4 Occurrence of multiple health risk factors and of each behavioral health risk factor profile stratified by school education (lowest, medium, highest) and employment status

\begin{tabular}{|c|c|c|c|c|c|c|c|c|c|c|c|c|}
\hline \multirow{2}{*}{$\begin{array}{l}\text { Health risk factor(s) } \\
\text { Mean number (standard deviation) }\end{array}$} & \multicolumn{2}{|c|}{$\begin{array}{l}\text { Lowest } \\
(n=1354)\end{array}$} & \multicolumn{2}{|c|}{$\begin{array}{l}\text { Medium } \\
(n=3715)\end{array}$} & \multicolumn{2}{|c|}{$\begin{array}{l}\text { Highest } \\
(n=1137)\end{array}$} & \multicolumn{2}{|c|}{$\begin{array}{l}\text { Unemployed } \\
(n=749)\end{array}$} & \multicolumn{2}{|c|}{$\begin{array}{l}\text { Employed } \\
(n=3680)\end{array}$} & \multicolumn{2}{|c|}{$\begin{array}{l}\text { Others } \\
(n=1791)\end{array}$} \\
\hline & 2.2 & $(0.9)$ & 1.9 & $(0.9)$ & 1.5 & (1.0) & 2.2 & $(0.9)$ & 1.8 & $(1.0)$ & 1.8 & $(0.9)$ \\
\hline & $\%$ & $(95 \%$ CI) & $\%$ & $(95 \%$ CI) & $\%$ & (95\% Cl) & $\%$ & $(95 \%$ CI) & $\%$ & $(95 \%$ CI) & $\%$ & $(95 \%$ CI) \\
\hline Multiple, $\geq 2$ & 79.0 & $(76.8-81.2)$ & 66.5 & $(64.9-68.0)$ & 48.0 & $(45.1-51.0)$ & 77.8 & $(74.7-80.8)$ & 63.6 & $(62.0-65.1)$ & 65.3 & $(63.1-67.5)$ \\
\hline \multicolumn{13}{|l|}{ Profile } \\
\hline no health risk factor & 2.3 & $(1.6-3.2)$ & 7.0 & $(6.2-7.8)$ & 16.9 & $(14.8-19.2)$ & 2.1 & $(1.2-3.4)$ & 9.3 & $(8.4-10.3)$ & 6.9 & $(5.7-8.1)$ \\
\hline smoking (only) & 3.7 & $(2.8-4.8)$ & 3.6 & $(3.0-4.3)$ & 4.0 & $(3.0-5.4)$ & 2.8 & $(1.7-4.3)$ & 4.0 & $(3.4-4.6)$ & 3.6 & $(2.8-4.6)$ \\
\hline alcohol (only) & 0.5 & $(0.2-1.1)$ & 0.8 & $(0.5-1.2)$ & 4.7 & $(3.5-6.1)$ & 0.9 & $(0.4-1.9)$ & 1.7 & $(1.3-2.2)$ & 1.2 & $(0.7-1.8)$ \\
\hline overweight (only) & 7.3 & $(6.0-8.8)$ & 12.3 & $(11.2-13.4)$ & 14.2 & $(12.2-16.3)$ & 8.5 & $(6.6-10.8)$ & 12.5 & $(11.5-13.6)$ & 10.7 & $(9.3-12.2)$ \\
\hline inactivity (only) & 7.2 & $(5.8-8.7)$ & 9.9 & $(8.9-10.9)$ & 12.2 & $(10.4-14.3)$ & 7.7 & $(5.9-9.9)$ & 8.9 & $(8.0-9.9)$ & 12.3 & $(10.9-14.0)$ \\
\hline smoking plus alcohol & 1.7 & $(1.1-2.5)$ & 1.2 & $(0.9-1.6)$ & 4.4 & $(3.3-5.8)$ & 2.4 & $(1.4-3.8)$ & 1.8 & $(1.4-2.3)$ & 1.8 & $(1.2-2.5)$ \\
\hline smoking plus overweight & 3.2 & $(2.4-4.3)$ & 4.6 & $(3.9-5.3)$ & 3.1 & $(2.2-4.3)$ & 3.9 & $(2.6-5.5)$ & 4.5 & $(3.86-5.2)$ & 3.0 & $(2.3-3.92)$ \\
\hline smoking plus inactivity & 11.9 & $(10.2-13.7)$ & 9.0 & $(8.1-10.0)$ & 4.3 & $(3.2-5.7)$ & 10.8 & $(8.7-13.3)$ & 8.1 & $(7.2-9.0)$ & 9.5 & $(8.2-10.9)$ \\
\hline alcohol plus overweight & 1.5 & $(0.9-2.3)$ & 2.5 & $(2.0-3.1)$ & 3.7 & $(2.7-5.0)$ & 1.9 & $(1.0-3.1)$ & 2.9 & $(2.4-3.5)$ & 1.8 & $(1.3-2.6)$ \\
\hline alcohol plus inactivity & 0.8 & $(0.4-1.4)$ & 1.3 & $(1.0-1.7)$ & 2.3 & $(1.5-3.3)$ & 1.6 & $(0.8-2.8)$ & 1.5 & $(1.2-2.0)$ & 0.9 & $(0.5-1.4)$ \\
\hline overweight plus inactivity & 25.3 & $(23.0-27.7)$ & 23.3 & $(22.0-24.7)$ & 16.3 & $(14.2-18.5)$ & 20.4 & $(17.6-23.5)$ & 20.8 & $(19.5-22.2)$ & 26.7 & $(24.7-28.9)$ \\
\hline smoking plus alcohol plus overweight & 1.6 & $(1.0-2.4)$ & 1.5 & $(1.1-1.9)$ & 1.7 & $(1.0-2.6)$ & 1.7 & $(0.9-2.9)$ & 1.7 & $(1.3-2.2)$ & 1.1 & $(0.6-1.7)$ \\
\hline smoking plus alcohol plus inactivity & 6.3 & $(5.0-7.7)$ & 4.0 & $(3.4-4.7)$ & 2.7 & $(1.9-3.8)$ & 8.8 & $(6.9-11.1)$ & 3.6 & $(3.0-4.2)$ & 3.8 & $(3.0-4.8)$ \\
\hline $\begin{array}{l}\text { smoking plus overweight plus } \\
\text { inactivity }\end{array}$ & 16.8 & $(14.8-18.9)$ & 10.6 & $(9.6-11.6)$ & 4.3 & $(3.2-5.7)$ & 14.8 & $(12.4-17.6)$ & 9.7 & $(8.8-10.7)$ & 11.3 & $(9.9-12.8)$ \\
\hline alcohol plus overweight plus inactivity & 4.1 & $(3.1-5.3)$ & 4.6 & $(3.9-5.3)$ & 3.3 & $(2.3-4.5)$ & 4.5 & $(3.2-6.3)$ & 4.8 & $(4.1-5.6)$ & 2.8 & $(2.1-3.7)$ \\
\hline all four health risk factors & 5.8 & $(4.6-7.2)$ & 3.9 & $(3.3-4.5)$ & 2.0 & $(1.3-3.0)$ & 6.9 & $(5.2-9.0)$ & 4.0 & $(3.4-4.7)$ & 2.6 & $(1.9-3.4)$ \\
\hline
\end{tabular}

Notes: $\mathrm{Cl}=$ confidence interval, non-overlapping $95 \% \mathrm{Cls}$ indicate significant differences at $\mathrm{p} \leq 0.01$

\section{Multivariate analysis on the number of HRFs}

As depicted in Table 5, the multivariate analysis revealed that the number of HRFs was significantly increased in males (incident rate ratio, IRR $=1.18$ ), 35-49 year olds $(I R R=1.13)$, 50-64 year olds $(I R R=1.07)$, persons with the lowest $(\mathrm{IRR}=1.39)$ or medium $(\mathrm{IRR}=1.23)$ level of school education, and unemployed persons (IRR $=1.12$ ). No significantly increased IRRs were found for medical department.

\section{Discussion}

So far, little is known about HRF profiles in general hospital patients, and this study helps to fill an important gap of knowledge. Five key findings emerged from the study: 1) Regardless of medical department, two thirds of all patients reported multiple behavioral HRFs. 2) While overall the most common HRF profiles involved energy-balance behaviors, trauma surgery and ear-nosethroat patients had particularly increased rates of substance-use related HRF profiles. 3) Men had higher rates than women concerning almost all HRF profiles involving multiple HRFs and alcohol. 4) While older patients had higher rates of HRF profiles involving multiple and both energy-balance related HRFs, younger patients had higher rates of HRF profiles involving at least one substance-use related HRF. 5) A social gradient was found: The lower the school education, the riskier the HRF profiles.

Compared to the general population, the study revealed similar proportions of the single HRFs alcohol, overweight and physical inactivity among general hospital patients [11]. However, markedly larger proportions of current smokers (39\% versus $28 \%$ ) and of persons with two or more HRFs were found (66\% versus $55 \%$, 11 ) indicating that needs in terms of behavior change interventions are particularly complex in general hospital patients. This appears to be plausible as hospital patients may have been admitted for reasons attributable to HRFs [1, 2], and as co-occurring HRFs can have more than additive effects on disease incidence (e.g. 17, 18). However, in addition to the fact that general hospital patients may be considered to be more morbid than the general population, the differences and similarities noted may be under- or overestimated due to different sample characteristics resulting from different inclusion criteria. For example, this study's sample that included adult 
Table 5 Multivariate poisson regression analysis predicting the number of reported health risk factors $(n=6205)$

\begin{tabular}{|c|c|c|c|c|}
\hline Predictors & & incident rate ratio & $95 \% \mathrm{Cl}$ & $\mathrm{p}$ \\
\hline \multirow[t]{3}{*}{ Medical department (internal medicine*) } & General surgery & 0.99 & $0.93-1.04$ & 0.638 \\
\hline & Ear-nose-throat & 1.02 & $0.97-1.07$ & 0.430 \\
\hline & Trauma surgery & 1.01 & $0.96-1.06$ & 0.655 \\
\hline Gender (female*) & Male & 1.18 & $1.14-1.23$ & $<0.001$ \\
\hline \multirow[t]{2}{*}{ Age group (18-34 years*) } & $35-49$ years & 1.13 & $1.07-1.19$ & $<0.001$ \\
\hline & 50-64 years & 1.07 & $1.02-1.12$ & 0.01 \\
\hline \multirow[t]{2}{*}{ Achieved school level (highest*) } & Lowest & 1.39 & $1.31-1.48$ & $<0.001$ \\
\hline & Medium & 1.23 & $1.16-1.30$ & $<0.001$ \\
\hline \multirow[t]{2}{*}{ Employment status (employed*) } & Unemployed & 1.12 & $1.06-1.19$ & $<0.001$ \\
\hline & Other & 0.99 & $0.95-1.04$ & 0.677 \\
\hline
\end{tabular}

Notes: * reference, $\mathrm{Cl}=$ confidence interval

patients aged 18-64 years regardless of living situation, was younger (mean age of 46 versus 60 years), included fewer females ( $41 \%$ versus $52 \%$ ), and fewer persons with the lowest level of school (22\% versus $32 \%$ ) than the German general population sample that included adults living in a private household with landline phone numbers regardless of age [11]. Nevertheless, the large proportion of general hospital patients with two or more HRFs shows a particular high need of preventive measures simultaneously targeting multiple HRFs in the general hospital setting.

Energy-balance related HRF profiles were particularly common in the total sample. With a proportion of $22 \%$, overweight plus inactivity was the most common HRF profile as also found in a general population and in a general hospital study in Germany [11, 44]. Similar to a previous general hospital study that investigated the HRFs smoking and alcohol only [57], our findings showed that about half of the hospital patients reported at least one of the two substance-use related HRFs. The current study also revealed that the occurrence of energy-balance HRF profiles was even larger in internal medicine and general surgery patients, while substanceuse related HRF profiles were more common in earnose-throat and trauma surgery patients. The different relevance of various HRF profiles in different medical departments is plausible. For example, joint at-risk alcohol use and tobacco smoking has more than a multiplicative effect on risk of head and neck cancers [18] which are typically treated in ear-nose-throat departments. Although differences in proportional relevance need to be considered when providing preventive measures, our findings also indicate that they should not be limited to single HRFs or HRF profiles as all four HRFs were common in all departments investigated.

In line with previous studies, gender, age and socioeconomic subgroups differed concerning the occurrence of any and multiple HRFs, but also concerning single HRF profiles (e.g. 11, 19). Among male, older, lower educated, and unemployed patients, larger proportions of any and multiple HRFs were found compared to their respective counterparts. The magnitude of difference was particularly large concerning education: $79 \%$ of the patients with the lowest versus $48 \%$ of the patients with the highest level of school reported multiple HRFs. There was a clear social gradient concerning both SES-related measures: The lower the level of school was, the higher were the proportions of patients with any HRFs, two or more HRFs, and all four HRFs. In addition, unemployment was also a significant and independent predictor of the accumulation of HRFs. Recent studies have shown that social inequalities with regards to the accumulation of HRFs have rather increased over the years [58]. This may also explain the increased social disparities in adult mortality in some regions of developed countries [59]. Our findings support the necessity of behavior change interventions to aim at closing the gap or (at least) at preventing a further widening of the gap by achieving greater reach and greater effectiveness in low SES groups [60]. For example, given that duration of unemployment has been shown to be related to the accumulation of HRFs [61], providing screening and brief intervention at job-agencies could be a feasible and effective approach as found concerning reduced alcohol use [62].

In addition to subgroup differences concerning the number of HRFs, subgroups also differed concerning HRF profiles. Overall, female, older, lower educated, and employed patients tended to show energy-balance related HRF profiles; and male, younger, higher educated, and unemployed patients tended to show substance-use related HRF profiles more often than their respective counterparts. However, while in some subgroup comparisons, profiles involving certain HRFs occurred more frequently in one group, riskier profiles involving the same HRF occurred more frequently in the comparison group. In particular, the rates of HRF profiles involving at-risk 
alcohol use were higher in patients with the highest compared to those with the lowest level of school. This fits in with findings from general population studies revealing higher proportions of alcohol drinkers and higher consumption scores with higher education and higher SES (e.g. [63, 64]). However, also in line with previous research [19], the patients with the lowest level of school more often reported riskier HRF profiles involving alcohol, namely profiles that involved multiple other HRFs in addition to at-risk alcohol use. Similarly, women clearly showed larger proportions of three of the most common HRF profiles, all including inactivity with a maximum of one more HRF. However, in line with previous findings [19], men more often reported those HRF profiles that included inactivity plus two or three more HRFs. Brief interventions targeting multiple HRFs need to consider this.

The strengths of this study include: The sample included a large proportion of all eligible patients (92\%). It may also be considered representative of general hospital patients treated for various diseases and injuries at four major general hospital departments. We investigated not only the occurrence but also the co-occurrence of four behavioral HRFs known as major contributors to the development and maintenance of non-communicable diseases in general hospital patients. Our study delineates differences in HRFs between different disciplines treating patients. It investigated unemployment as an independent predictor of the accumulation of HRFs in addition to school education.

Several limitations of the study need to be considered. The first limitation concerns the possible underestimation of the proportions of HRFs. The assessment was based on self-report and might be distorted by patients' tendencies to provide socially desirable answers. For example, self-reported statements as used in our study are likely to underestimate overweight [65]. The inactivity measure used may have underestimated either physical inactivity or physical activity. Although HRF definitions were based on current recommendations $[2,6]$, the measure provided a rather rough and limited estimation of physical activity. For example, it assessed sports activity only rather than in combination with job-, transportand housework-related physical activity as done by the international physical activity questionnaire [66]; and it did not differentiate intensity levels of physical activity for which separate recommendations exist, e.g. at least $75 \mathrm{~min}$ of vigorous- or $150 \mathrm{~min}$ of moderate-intensity physical activity per week $[54,55]$. Due to item restrictions, and to prevent misclassification of patients with vigorous-intensity activity, a more liberal cut-off than recommended, i.e. less than 1 hour of sports per week was applied to determine physical inactivity. Given that over-reporting of physical activity is a common problem, also when assessed by more established questionnaires [67], and as physical activity was not in the focus of the original trial which focused on alcohol [49], a simple measure was preferred to keep the screening as brief as possible. Furthermore, HRFs on general surgery wards may have been underestimated as many surgical patients present on internal medicine wards first, and this study only assessed data during their first hospital admission. The second limitation concerns the generalizability of our findings to patients from other departments, or to other general hospitals in Germany or beyond. A multisite study in Germany, however, revealed similar proportions of the single HRFs [44]. The third limitation concerns that some significant differences in proportions between subgroups may have been missed. By interpreting non-overlapping 95\% confidence intervals (a common procedure in epidemiological research), differences at about $p=0.01$ and smaller may be found, but differences at about $p>0.01$ to $p<0.05$ may remain undetected [56]. However, to avoid multiple testing and inflation of $p$-values, additional test statistics were not calculated in this study. The final limitation concerns the lack of information on the medical condition, costs of treatment and length of hospital stay. It may be expected that the co-occurrence of HRFs and the occurrence of particular HRF profiles may be related to higher health care costs. Future research should investigate this.

\section{Conclusions}

Given that a lower number of behavioral HRFs is related to a lower risk of mortality $[15,16]$, and two thirds of the patients reporting profiles involving multiple HRFs, our study showed a high need for systematic screening and intervention targeting multiple HRF in general hospital patients. As health care still lacks systematic preventive measures accessible to all patients, the potential for the prevention and treatment of non-communicable diseases is being missed.

Systematically identifying and addressing patients' individual HRF profiles may have a great potential for at least two reasons: Firstly, it may simultaneously serve primary and secondary prevention purposes by preventing the onset of chronic diseases in patients who have not been diagnosed with chronic diseases yet, and by improving treatment success and prognosis in patients with chronic diseases. Although a large part of the general hospital patients is expected to have been diagnosed with chronic diseases in the past, our study supports previous findings showing that routine health care alone may not be sufficient for patients with chronic diseases to change their lifestyle [68, 69]. Secondly, systematic multiple HRF screening and intervention may provide the means to reach most patients, including those most in need, namely male, older, lower educated, and 
unemployed patients as indicated by our findings. These subgroups, and low SES people in particular have been found to be particularly hard to reach otherwise [70].

Medical care staff or health behavior change interventionists should be prepared to screen for and address multiple HRFs in each patient. With high reach of a systematic multiple HRF screening and efficacy of single HRF interventions [29-37], a multiple HRF approach is likely to have clinical and public health impact [71, 72]. Future research and implementation research in particular should investigate this.

\section{Abbreviations}

Cl: Confidence interval; HRF: Health risk factor; M: Mean; PECO: Randomized controlled trial "Testing delivery channels of individualized motivationally tailored alcohol interventions among general hospital patients: in-person versus computer-based"; SD: Standard deviation; SES: Socio-economic status

\section{Acknowledgements}

We appreciate Elke Bandelin, Kornelia Sadewasser and Stefanie Tobschall for recruiting the participants and Christian Goeze for programming the screening software. We are grateful for the support provided by the heads and staff of the participating departments at the University Medicine Hospital Greifswald.

\section{Authors' contributions}

JFA contributed to the conception and design of the work, acquisition, analysis and interpretation of data, and drafted the work. FN contributed to the analysis and interpretation of data. SB contributed to the acquisition and interpretation of data. AAA and USM contributed to the interpretation of data. BG and UJ contributed to the conception and design of the work, and interpretation of data. All authors have approved the submitted version; and have agreed both to be personally accountable for the author's own contributions and to ensure that questions related to the accuracy or integrity of any part of the work, even ones in which the author was not personally involved, are appropriately investigated, resolved, and the resolution documented in the literature.

\section{Funding}

The study was funded by grants from the German Cancer Aid to JFA, BG and UJ (108376, 109737, 110676, 110543, 111346), and to JFA and UJ (70110543). We acknowledge support for the Article Processing Charge from the German Research Foundation (DFG, 393148499) and the Open Access Publication Fund of the University of Greifswald. The funders had no role in study design, data collection and analysis, decision to publish, or preparation of the manuscript.

\section{Availability of data and materials}

The datasets generated and analyzed during the current study are not publicly available due to the German data protection law but are available from the corresponding author on reasonable request that complies with the study purpose and the participants informed consent.

\section{Ethics approval and consent to participate}

The ethics committee of the University Medicine Greifswald approved the study procedure $(\mathrm{BB} 07 / 10)$. As approved by the ethics committee, the screening participants whose data were evaluated as part of this study provided informed oral consent. Participants eligible to participate in the subsequent randomized controlled trial provided informed written consent.

\section{Consent for publication}

Not applicable.

\section{Competing interests}

The authors declare that they have no competing interests.

\section{Author details}

${ }^{1}$ Institute for Medical Psychology, University Medicine Greifswald, Walther-Rathenau-Str. 48, 17475 Greifswald, Germany. ${ }^{2}$ German Center for Cardiovascular Research, Site Greifswald, Fleischmannstr. 42-44, 17475 Greifswald, Germany. ${ }^{3}$ Clinic and Policlinic of Urology, University Medicine Greifswald, Ferdinand-Sauerbruch-Straße, 17475 Greifswald, Germany. ${ }^{4}$ Institute of Social Medicine and Prevention, University Medicine Greifswald, Walther-Rathenau-Str. 48, 17475 Greifswald, Germany. ${ }^{5}$ Institute and Policlinic of Occupational and Social Medicine, Faculty of Medicine, Technische Universität Dresden, Fetscherstraße 74, 01307 Dresden, Germany. ${ }^{6}$ Department of Internal Medicine A, University Medicine Greifswald, Ferdinand-Sauerbruch-Straße, 17475 Greifswald, Germany. 'Department of Epidemiology and Health Monitoring, Robert Koch Institute Berlin, General-Pape-Str. 62-66, 12101 Berlin, Germany.

Received: 8 February 2019 Accepted: 8 November 2019

Published online: 29 November 2019

\section{References}

1. Risk Factors Collaborators GBD. Global, regional, and national comparative risk assessment of 84 behavioural, environmental and occupational, and metabolic risks or clusters of risks, 1990-2016: a systematic analysis for the global burden of disease study 2016. Lancet. 2017;390(10100):1345-422.

2. World Health Organization. Global status report on noncommunicable diseases 2014. Geneva: WHO Press; 2014.

3. Mokdad AH, Marks JS, Stroup DF, Gerberding JL. Actual causes of death in the United States, 2000. JAMA. 2004;291(10):1238-45.

4. Lei YY, Ho SC, Cheng A, Kwok C, Lee Cl, Cheung KL, et al. Adherence to the World Cancer Research Fund/American Institute for Cancer Research guideline is associated with better health-related quality of life among Chinese patients with breast Cancer. J Natl Compr Cancer Netw. 2018;16(3):275-85.

5. Braithwaite D, Izano M, Moore DH, Kwan ML, Tammemagi MC, Hiatt RA, et al. Smoking and survival after breast cancer diagnosis: a prospective observational study and systematic review. Breast Cancer Res Treat. 2012; 136(2):521-33.

6. World Cancer Research Fund/American Institute for Cancer Research. Diet, Nutrition, Physical Activity and Cancer: a Global Perspective. Continous Update Project Expert Report 2018. Washington: World Cancer Research Fund/American Institute for Cancer Research; 2018.

7. World Cancer Research Fund/American Institute for Cancer Research. Continous Update Project Expert Report 2018. In: Survivors of breast and other cancers. Washington: World Cancer Research Fund/American Institute for Cancer Research; 2018.

8. American Heart Association. The American Heart Association Diet and Lifestyle Recommendations 2019 [Available from: https://www.heart.org/en/ healthy-living/healthy-eating/eat-smart/nutrition-basics/aha-diet-andlifestyle-recommendations, accessed on 14 June 2019.

9. Schnuerer I, Baumann S, Haberecht K, Gaertner B, John U, Freyer-Adam J. Patterns of health risk behaviors among job-seekers: a latent class analysis. Int J Public Health. 2015;60(1):111-9.

10. de Vries H. Van 't Riet J, Spigt M, Metsemakers J, van den Akker M, Vermunt $J K$, et al. clusters of lifestyle behaviors: results from the Dutch SMILE study. Prev Med. 2008;46(3):203-8.

11. John U, Hanke M, Freyer-Adam J. Health Risk Behavior Patterns in a National Adult Population Survey. Int J Environ Res Public Health. 2018;15(5).

12. Schuit AJ, van Loon AJ, Tijhuis M, Ocke M. Clustering of lifestyle risk factors in a general adult population. Prev Med. 2002;35(3):219-24

13. Poortinga $\mathbf{W}$. The prevalence and clustering of four major lifestyle risk factors in an English adult population. Prev Med. 2007:44(2):124-8.

14. Liu Y, Croft JB, Wheaton AG, Kanny D, Cunningham TJ, Lu H, et al. Clustering of five health-related behaviors for chronic disease prevention among adults, United States, 2013. Prev Chronic Dis. 2016;13:E70.

15. Khaw KT, Wareham N, Bingham S, Welch A, Luben R, Day N. Combined impact of health behaviours and mortality in men and women: the EPICNorfolk prospective population study. PLoS Med. 2008;5(1):e12.

16. Petersen KE, Johnsen NF, Olsen A, Albieri V, Olsen LK, Dragsted LO, et al. The combined impact of adherence to five lifestyle factors on all-cause, cancer and cardiovascular mortality: a prospective cohort study among Danish men and women. Br J Nutr. 2015;113(5):849-58.

17. Castellsague X, Munoz N, De Stefani E, Victora CG, Castelletto R, Rolon PA, et al. Independent and joint effects of tobacco smoking and alcohol 
drinking on the risk of esophageal cancer in men and women. Int J Cancer. 1999:82(5):657-64.

18. Hashibe M, Brennan P, Chuang SC, Boccia S, Castellsague X, Chen C, et al. Interaction between tobacco and alcohol use and the risk of head and neck cancer: pooled analysis in the international head and neck Cancer epidemiology consortium. Cancer Epidemiol Biomark Prev. 2009;18(2):541-50.

19. Noble N, Paul C, Turon H, Oldmeadow C. Which modifiable health risk behaviours are related? A systematic review of the clustering of smoking, nutrition, alcohol and physical activity ('SNAP') health risk factors. Prev Med. 2015;81:16-41

20. Mackenbach JP, Stirbu I, Roskam AJ, Schaap MM, Menvielle G, Leinsalu M, et al. Socioeconomic inequalities in health in 22 European countries. N Engl J Med. 2008;358(23):2468-81.

21. Mackenbach JP, Bopp M, Deboosere P, Kovacs K, Leinsalu M, Martikainen P, et al. Determinants of the magnitude of socioeconomic inequalities in mortality: a study of 17 European countries. Health Place. 2017:47:44-53.

22. Morris L, D'Este C, Sargent-Cox K, Anstey KJ. Concurrent lifestyle risk factors: clusters and determinants in an Australian sample. Prev Med. 2016;84:1-5.

23. Thebault JL, Ringa V, Panjo H, Bloy G, Falcoff H, Rigal L. Accumulation of unhealthy behaviors: marked social inequalities in men and women. Prev Med Rep. 2018;12:1-5.

24. Longabaugh R, Minugh PA, Nirenberg TD, Clifford PR, Becker B, Woolard R. Injury as a motivator to reduce drinking. Acad Emerg Med. 1995;2(9):817-25.

25. McBride CM, Emmons KM, Lipkus IM. Understanding the potential of teachable moments: the case of smoking cessation. Health Educ Res. 2003;18(2):156-70.

26. Emmons KM, Goldstein MG. Smokers who are hospitalized: a window of opportunity for cessation interventions. Prev Med. 1992;21(2):262-9.

27. Rumpf H-J, Hapke U, Meyer C, John U. Motivation to change drinking behavior: comparison of alcohol-dependent individuals in a general hospital and a general population sample. Gen Hosp Psychiatry. 1999;21:348-53.

28. Haynes CL. Health promotion services for lifestyle development within a UK hospital--Patients' experiences and views. BMC Public Health. 2008;8:284

29. Bully P, Sanchez A, Zabaleta-Del-Olmo E, Pombo H, Grandes G. Evidence from interventions based on theoretical models for lifestyle modification (physical activity, diet, alcohol and tobacco use) in primary care settings: a systematic review. Prev Med. 2015;765:S76-93.

30. Kaner EF, Beyer FR, Muirhead C, Campbell F, Pienaar ED, Bertholet N, et al. Effectiveness of brief alcohol interventions in primary care populations. Cochrane Database Syst Rev. 2018;2:CD004148.

31. Lundahl B, Moleni T, Burke BL, Butters R, Tollefson D, Butler C, et al. Motivational interviewing in medical care settings: a systematic review and meta-analysis of randomized controlled trials. Patient Educ Couns. 2013; 93(2):157-68.

32. Mdege ND, Fayter D, Watson JM, Stirk L, Sowden A, Godfrey C. Interventions for reducing alcohol consumption among general hospital inpatient heavy alcohol users: a systematic review. Drug Alcohol Depend. 2013;131(1-2):1-22.

33. Nair NK, Newton NC, Shakeshaft A, Wallace P, Teesson M. A systematic review of digital and computer-based alcohol intervention programs in primary care. Curr Drug Abuse Rev. 2015;8(2):111-8.

34. O'Donnell A, Anderson P, Newbury-Birch D, Schulte B, Schmidt C, Reimer J, et al. The impact of brief alcohol interventions in primary healthcare: a systematic review of reviews. Alcohol Alcohol. 2014;49(1):66-78.

35. Orrow G, Kinmonth AL, Sanderson S, Sutton S. Effectiveness of physical activity promotion based in primary care: systematic review and metaanalysis of randomised controlled trials. BMJ. 2012;344:e1389.

36. Sargent GM, Forrest LE, Parker RM. Nurse delivered lifestyle interventions in primary health care to treat chronic disease risk factors associated with obesity: a systematic review. Obes Rev. 2012;13(12):1148-71.

37. VanBuskirk KA, Wetherell JL. Motivational interviewing with primany care populations: a systematic review and meta-analysis. J Behav Med. 2014;37(4):768-80.

38. Freyer-Adam J, Baumann S, Haberecht K, Bischof G, Meyer C, Rumpf HJ, et al. Can brief alcohol interventions in general hospital inpatients improve mental and general health over 2 years? Results from a randomized controlled trial. Psychol Med. 2019:49(10):1722-30.

39. Funderburk JS, Maisto SA, Sugarman DE, Wade M. The covariation of multiple risk factors in primary care: a latent class analysis. J Behav Med. 2008;31(6):525-35.

40. Baumann S, Toft U, Aadahl M, Jorgensen T, Pisinger C. The long-term effect of screening and lifestyle counseling on changes in physical activity and diet: the Inter99 study - a randomized controlled trial. Int J Behav Nutr Phys Act. 2015;12:33.

41. Baumann S, Toft U, Aadahl M, Jorgensen T, Pisinger $C$. The long-term effect of a population-based life-style intervention on smoking and alcohol consumption. The Inter99 study--a randomized controlled trial. Addiction. 2015;110(11):1853-60.

42. Parekh S, King D, Boyle FM, Vandelanotte C. Randomized controlled trial of a computer-tailored multiple health behaviour intervention in general practice: 12-month follow-up results. Int I Behav Nutr Phys Act. 2014;11(1):41.

43. Schulz DN, Kremers SP, Vandelanotte C, van Adrichem MJ, Schneider F, Candel $\mathrm{MJ}$, et al. Effects of a web-based tailored multiple-lifestyle intervention for adults: a two-year randomized controlled trial comparing sequential and simultaneous delivery modes. J Med Internet Res. 2014;16(1):e26.

44. Guertler D, Moehring A, Krause K, Eck S, Batra A, Chenot JF, et al. Proactive multipurpose health risk screening in health care settings: Methods, design, and reach. Int J Methods Psychiatr Res. 2019:e1760.

45. Freyer-Adam J, Baumann S, Haberecht K, Tobschall S, Schnuerer I, Bruss K, et al. In-person and computer-based alcohol interventions at general hospitals: reach and retention. Eur J Pub Health. 2016;26(5):844-9.

46. Freyer-Adam J, Baumann S, Schnuerer I, Haberecht K, John U, Gaertner B. [In-person vs. computer-based alcohol intervention for general hospital inpatients: Study design] Sucht. 2015;61(6):347-55.

47. Baumann S, Gaertner B, Haberecht K, Bischof G, John U, Freyer-Adam J. How alcohol use problem severity affects the outcome of brief intervention delivered in-person versus through computer-generated feedback letters. Drug Alcohol Depend. 2018;183:82-8.

48. Baumann S, Gaertner B, Haberecht K, Meyer C, Rumpf HJ, John U, et al. Does impaired mental health interfere with the outcome of brief alcohol intervention at general hospitals? J Consult Clin Psychol. 2017;85(6):562-73.

49. Freyer-Adam J, Baumann S, Haberecht K, Tobschall S, Bischof G, John U, et al. In-person alcohol counseling versus computer-generated feedback: results from a randomized controlled trial. Health Psychol. 2018;37(1):70-80.

50. Bush K, Kivlahan DR, McDonell MB, Fihn SD, Bradley KA. The AUDIT alcohol consumption questions (AUDIT-C): an effective brief screening test for problem drinking. Arch Intern Med. 1998;158(16):1789-95.

51. Reinert DF, Allen JP. The alcohol use disorders identification test: an update of research findings. Alcohol Clin Exp Res. 2007;31(2):185-99.

52. Seitz HK, Bühringer G, Mann K. [Limits for the consumption of alcoholic beverages: Guidelines of the scientific board of trustees of the DHS]. In: DHS, editor. Yearbook Addiction 2008. Geesthacht: Neuland; 2008. p. 205-209.

53. WHO report obesity. Preventing and managing the global epidemic. Report of a WHO consultation on obesity. Genf: WHO; 1998.

54. Arnett DK, Blumenthal RS, Albert MA, Buroker AB, Goldberger ZD, Hahn EJ, et al. 2019 ACC/AHA guideline on the primary prevention of cardiovascular disease: a report of the American College of Cardiology/American Heart Association task force on clinical practice guidelines. Circulation. 2019; 140(11):e596-646.

55. World Health Organization. Global recommendations on physical activity for health. Geneva: WHO Press; 2010.

56. Cumming G. Inference by eye: reading the overlap of independent confidence intervals. Stat Med. 2009;28(2):205-20.

57. Coder B, Freyer-Adam J, Rumpf H-J, John U, Hapke U. Tobacco smoking and alcohol problem drinking among general hospital inpatients. Prävention und Gesundheitsförderung. 2008;3:37-42.

58. Ding D, Do A, Schmidt HM, Bauman AE. A widening gap? Changes in multiple lifestyle Risk Behaviours by socioeconomic status in New South Wales, Australia, 2002-2012. PLoS One. 2015;10(8):e0135338.

59. Montez JK, Zajacova A, Hayward MD, Woolf SH, Chapman D, Beckfield J. Educational disparities in adult mortality across U.S. states: how Do they differ, and have they changed since the mid-1980s? Demography. 2019;56(2):621-44.

60. Mackenbach JP, Kulhanova I, Artnik B, Bopp M, Borrell C, Clemens T, et al. Changes in mortality inequalities over two decades: register based study of European countries. BMJ. 2016;353:11732.

61. Freyer-Adam J, Gaertner B, Tobschall S, John U. Health risk factors and selfrated health among job-seekers. BMC Public Health. 2011;11:659.

62. Freyer-Adam J, Baumann S, Schnuerer I, Haberecht K, Bischof G, John U, et al. Does stage tailoring matter in brief alcohol interventions for jobseekers? A randomized controlled trial. Addiction. 2014;109(11):1845-56.

63. Beard E, Brown J, West R, Angus C, Brennan A, Holmes J, et al. Deconstructing the alcohol harm paradox: a population based survey of adults in England. PLoS One. 2016;11(9):e0160666. 
64. Grittner U, Kuntsche S, Gmel G, Bloomfield K. Alcohol consumption and social inequality at the individual and country levels--results from an international study. Eur J Pub Health. 2013;23(2):332-9.

65. John U, Hanke M, Grothues J, Thyrian JR. Validity of overweight and obesity in a nation based on self-report versus measurement device data. Eur J Clin Nutr. 2006;60(3):372-7.

66. Craig CL, Marshall AL, Sjostrom M, Bauman AE, Booth ML, Ainsworth BE, et al. International physical activity questionnaire: 12-country reliability and validity. Med Sci Sports Exerc. 2003;35(8):1381-95.

67. Prince SA, Adamo KB, Hamel ME, Hardt J, Connor Gorber S, Tremblay M. A comparison of direct versus self-report measures for assessing physical activity in adults: a systematic review. Int J Behav Nutr Phys Act. 2008;5:56.

68. Jazieh AR, Foraida M, Ghouse M, Khalil MM, Kopp M, Savidge M. The impact of cancer diagnosis on the lifestyle and habits of patients served at a veterans administration hospital. J Cancer Educ. 2006;21(3):147-50

69. Tromp DM, Brouha XD, Hordijk GJ, Winnubst JA, Gebhardt WA, van der Doef MP, et al. Medical care-seeking and health-risk behavior in patients with head and neck cancer: the role of health value, control beliefs and psychological distress. Health Educ Res. 2005;20(6):665-75.

70. Bender AM, Jorgensen $T$, Helbech B, Linneberg A, Pisinger C. Socioeconomic position and participation in baseline and follow-up visits: the Inter99 study. Eur J Prev Cardiol. 2012;21(7):899-905.

71. Glasgow RE, Estabrooks PE. Pragmatic applications of RE-AIM for health care initiatives in community and clinical settings. Prev Chronic Dis. 2018;15:E02.

72. Glasgow RE, Vogt TM, Boles SM. Evaluating the public health impact of health promotion interventions: the RE-AIM framework. Am J Public Health. 1999;89(9):1322-7.

\section{Publisher's Note}

Springer Nature remains neutral with regard to jurisdictional claims in published maps and institutional affiliations.

Ready to submit your research? Choose BMC and benefit from:

- fast, convenient online submission

- thorough peer review by experienced researchers in your field

- rapid publication on acceptance

- support for research data, including large and complex data types

- gold Open Access which fosters wider collaboration and increased citations

- maximum visibility for your research: over $100 \mathrm{M}$ website views per year

At $\mathrm{BMC}$, research is always in progress.

Learn more biomedcentral.com/submissions 